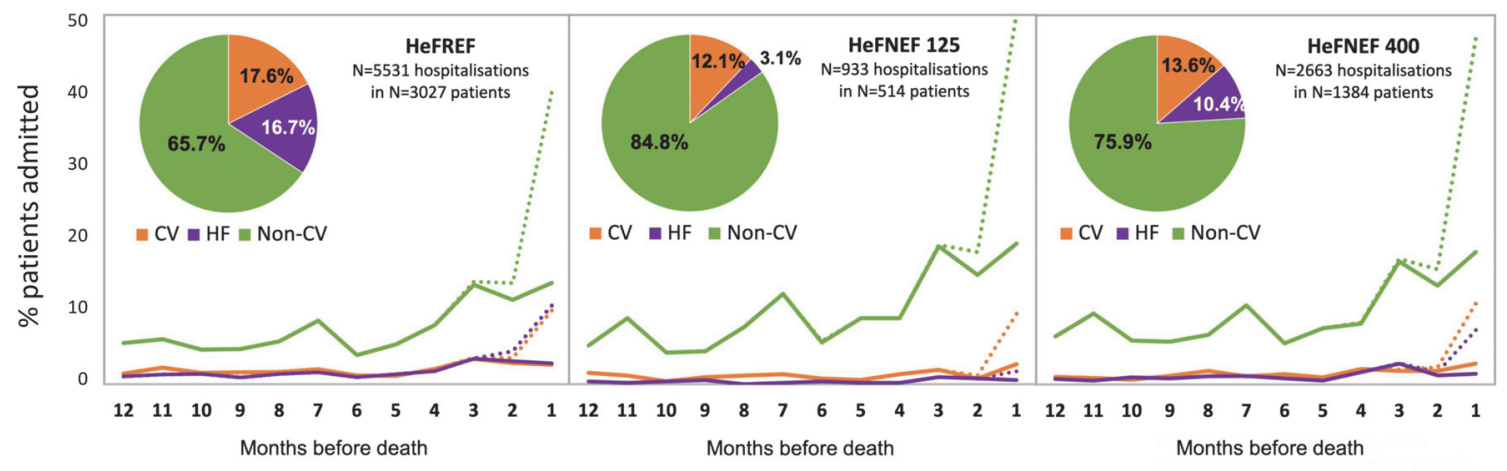

Bold lines: hospitalisations that resulted in discharge.

Dotted lines: hospitalisations where the patient died during admission.

Inset: Pie charts show the causes of admissions in the last year of life for each phenotype.

Abstract 120 Figure 1 Percentage of patients admitted each month prior to death

and pattern of hospitalisation for patients with $\mathrm{HF}$ in the 12 months preceding death.

Methods This is a retrospective analysis of patients recruited prospectively over two decades at a community HF clinic in Kingston upon Hull, UK. Patients with HF who died before August 2020 were included. We divided the cohort into three phenotypes: i) heart failure with reduced ejection fraction (HeFREF), ii) heart failure with normal ejection fraction (HeFNEF) with $\mathrm{N}$-terminal pro B-type natriuretic peptide (NTproBNP) 125-399 ng/L, and iii) HeFNEF with NTproBNP $\geq 400 \mathrm{ng} / \mathrm{L}$. The primary outcome was hospital admissions in the last year of life, classified as: HF, other cardiovascular (CV), or non-cardiovascular (non-CV). The primary cause of hospital admission using ICD-10 criteria was used.

Results Among 4925 patients, the median (Q1-Q3) age at death was 81 (75-87). $38 \%$ of patients were female. There were 9127 hospital admissions. The median (Q1-Q3) number of hospitalisations per patient was 2 (1-3) and days spent in hospital in the last year of life was 12 (2-25). 83\% of patients had at least one hospitalisation; $20 \%$ had at least one HF hospitalisation; 24\% had at least one CV hospitalisation; $70 \%$ had at least one non-CV hospitalisation. 54\% of deaths were as an in-patient. HF admissions were a minority in all phenotypes, but most common in HeFREF. In each group, at least two thirds of admissions were non-CV. $6 \%$ of the admissions in 2020 (prior to August) were for COVID-19. Hospitalisations increased in the three months prior to death (figure 1).

Conclusion In the last year of life in patients with HF, most hospitalisations are for non-CV causes regardless of $\mathrm{HF}$ phenotype.

Conflict of Interest None

\section{EFFECT OF UK COVID-19 PUBLIC HEALTH MEASURES ON ACTIVITY AND QUALITY OF LIFE IN PATIENTS WITH PULMONARY ARTERIAL HYPERTENSION}

${ }^{1}$ Jennifer Middleton, ${ }^{1}$ Hamza Zafar, ${ }^{2}$ Ashwin Reddy, ${ }^{3}$ Claire Martin, ${ }^{1}$ Roger Thompson, ${ }^{1}$ Andrew Swift, ${ }^{2}$ Mark Toshner, ${ }^{1}$ David.G Kiely, ${ }^{1}$ Alexander Rothman. ${ }^{1}$ University of Sheffield, Sheffield, UK; ${ }^{2}$ University of Cambridge; ${ }^{3}$ Royal Papworth NHS Foundation Trust

10.1136/heartjnl-2021-BCS.118

Background Limitation of activity and restriction of movement have been widely, and effectively, enforced to reduce COVID-
19 transmission. Physical activity is however a critical measure in the prevention of cardiovascular disease. Pulmonary arterial hypertension $(\mathrm{PAH})$ is a devastating, disease driven by small vessel vascular remodeling, leading to right heart failure. Exercise capacity relates to clinical outcomes and exercise training improves key indicators of cardiopulmonary function. Here, we describe the temporal effects of UK government restriction measures on daily activity, heart rate and quality-of-life (QoL) in patients with PAH.

Methods From November 2019 to March 2020 patients were enrolled into the arrhythmia sub-study of The UK National Cohort Study of Idiopathic and Heritable PAH (REC:13/EE/ 0203) and implanted with insertable cardiac monitors. Daily heart rate, heart rate variability and activity were transmitted remotely. Standard questionnaires were administered remotely to assess QoL (EmPHasis-10), anxiety (GAD-7) and depression (PHQ-9).

Results Median age of the 26 patients implanted with insertable cardiac monitors was 49 years, 23(88\%) were female and $5(19 \%)$ had heritable PAH with mutations in BMPR2. At enrolment $10(38.5 \%)$ patients were low risk $(<5 \%$ 1-year mortality), $10(38.5 \%)$ were intermediate risk $(5-10 \%)$ and 6 (23\%) were high risk (>10\%). The mean duration from insertion to census date was 21.1weeks \pm 5.7 . No complications were reported. Completeness of remote monitoring data was 100\%. Following lockdown, mean activity was reduced (3.16vrs 2.68hours, -0.48 hours, 95\%CI -0.27-0.69, 16\%, $\mathrm{p}<0.0001)$. During the period April 14th to 23rd QoL was reduced $(26(18-38)$ vrs $32(17-47), \mathrm{p}<0.01)$ and anxiety $(1(0-$ 9)vrs $10(5-18), \mathrm{p}<0.001)$ and depression scores increased (3 $(1-16)$ vrs 11 (3-17), p<0.001) compared to pre-lockdown levels. The observed increase in depression scores persisted to the census date $(3(1-16)$ vrs $11(8-17), \mathrm{p}<0.01)$. No change in day or night heart rate, or heart rate variability, was observed and no patients developed COVID-19.

Conclusion In this cohort of patients with idiopathic and heritable PAH, UK protective health measures were effective in preventing COVID-19 in patients thought to be vulnerable. However, these protective measures resulted in reduced daily activity and QoL and were associated increased anxiety and depression indicators. Patients may decondition through periods of reduced activity. This may have implications for riskassessment and endpoint adjudication in clinical studies, both of which use measures of exercise capacity.

Conflict of Interest none 